OPEN ACCESS

Edited by:

Sushant Sahastrabuddhe, International Vaccine Institute, South Korea

Reviewed by: Jaime Torres,

Central University of Venezuela, Venezuela

Yun-Chi Chen,

Morgan State University, United States

*Correspondence: James M. Fleckenstein jfleckenstein@wustl.edu

Specialty section:

This article was submitted to Vaccines for Tropical Diseases, a section of the journal

Frontiers in Tropical Diseases

Received: 14 May 2021 Accepted: 24 August 2021 Published: 14 September 2021

Citation: Fleckenstein JM (2021)

Confronting Challenges to Enterotoxigenic Escherichia coli Vaccine Development.

Front. Trop. Dis. 2:709907. doi: 10.3389/fitd.2021.709907

\section{Confronting Challenges to Enterotoxigenic Escherichia coli Vaccine Development}

\author{
James M. Fleckenstein ${ }^{1,2 *}$ \\ ${ }^{1}$ Department of Medicine, Division of Infectious Diseases, Washington University in Saint Louis, School of Medicine, Saint \\ Louis, MO, United States, ${ }^{2}$ Medicine Service, Infectious Diseases, John Cochran Saint Louis Veterans Affairs Health Care \\ System, Saint Louis, MO, United States
}

The enterotoxigenic Escherichia coli (ETEC) are a diverse and genetically plastic pathologic variant (pathovar) of $E$. coli defined by their production of heat-labile $(L T)$ and heat-stable (ST) enterotoxins. These pathogens, which came to recognition more than four decades ago in patients presenting with severe cholera-like diarrhea, are now known to cause hundreds of millions of cases of symptomatic infection annually. Children in lowmiddle income regions of the world lacking access to clean water and basic sanitation are disproportionately affected by ETEC. In addition to acute diarrheal morbidity, these pathogens remain a significant cause of mortality in children under the age of five years and have also been linked repeatedly to sequelae of childhood malnutrition and growth stunting. Vaccines that could prevent ETEC infections therefore remain a high priority. Despite several decades of effort, a licensed vaccine that protects against the breadth of these pathogens remains an aspirational goal, and the underlying genetic plasticity of $E$. coli has posed a fundamental challenge to development of a vaccine that can encompass the complete antigenic spectrum of ETEC. Nevertheless, novel strategies that include toxoids, a more complete understanding of ETEC molecular pathogenesis, structural details of target immunogens, and the discovery of more highly conserved antigens essential for virulence should accelerate progress and make a broadly protective vaccine feasible.

Keywords: adhesins, Escherichia coli, heat-labile toxin, vaccine, heat-stable toxin, diarrhea, global health, malnutrition

\section{THE ETEC DISEASE BURDEN AND THE NEED FOR A VACCINE}

Enterotoxigenic E. coli (ETEC) are ubiquitous pathogens in areas of the world where clean water and sanitation remain limited. Worldwide, ETEC are responsible for hundreds of millions of cases of diarrheal illness (1), disproportionately affecting young children under the age of five who have yet to become immune through prior exposure (2). ETEC remain a one of the principal causes of death due to diarrheal illness in young children in low-middle income countries (3). Although deaths from infectious diarrhea have declined over the past few decades due to introduction of oral rehydration and other measures, ETEC continue to exact a heavy toll in acute morbidity as well as associated sequelae that include malnutrition and growth stunting (4). Moreover, malnutrition 
linked to ETEC is also associated with excess mortality beyond direct deaths from acute diarrheal illness $(5,6)$. Ideally, an ETEC vaccine would prevent both the acute illness as well as the attendant sequelae.

Notably, while ETEC are more prevalent among young children in LMICs, they are not limited to any particular age group and can be found globally. These pathogens are also a major cause of diarrheal morbidity in older children and adults of LMICs (7), and perennially the most common etiology of diarrhea in travelers to LMICs. Moreover, both sporadic cases (8) as well as multiple large scale outbreaks (9-16) are welldocumented in the United States and other industrialized regions demonstrating that even where clean water and sanitation are widely available these organisms can be problematic.

Accurate estimation of ETEC disease burden is critical to future development of vaccines, as these estimates underly calculation of value assessments by the WHO and other agencies in prioritization of vaccines most likely to have a sustained impact on public health. Although, heterogeneity of disease burden estimates (1, 4, 17-19), can significantly confound cost-effectiveness estimates fundamental to setting vaccine development priorities, ETEC vaccines remain high priority targets of advisory groups including the World Health Organization (WHO) Product Development Vaccines Advisory Committee (PDVAC) (20).

\section{ETEC MOLECULAR PATHOGENESIS}

\subsection{Classical Paradigm for ETEC Pathogenesis}

The discovery of toxin-producing E. coli in patients presenting with clinical cholera-like diarrheal illness (21-23) soon led to the discovery of the responsible cholera toxin-like heat-labile toxin (LT) and heat-stable (ST) enterotoxins as well as the first plasmid-encoded fimbrial antigens, known as colonization factors (CFs) (24). Early controlled human infection studies demonstrated that a plasmid-cured version of ETEC (H10407-P) was avirulent when compared its parent (H10407) provided additional validation of the importance of CFs (25). From these early discoveries a model of ETEC pathogenesis evolved in which ETEC colonized the small intestine using CFs to support the delivery of enterotoxins that drove fluid export, and diarrhea. Although to date, a wide variety of plasmid encoded CFs and colonization surface (CS) antigens (more than 25 to date) have been discovered, the basic archetypical model of ETEC molecular pathogenesis stood.

\subsection{Contribution of Noncanonical Antigens to ETEC Virulence}

A potential pitfall of this limited view of virulence is that it potentially constrains ETEC vaccinology around a subset of canonical antigens, the CFs and toxins. A number of recent studies suggest that this classical paradigm for ETEC molecular pathogenesis is incomplete, and that additional molecules could potentially be targeted to expand and complement the existing antigenic repertoire.

Two plasmid-encoded antigens identified in the course of studies to identify novel molecules that are either surfaceexpressed or secreted by ETEC, now appear to play important roles in molecular pathogenesis. Both molecules are relatively conserved within the ETEC pathovar, potentially attesting to their viability as vaccine targets and their importance for virulence. Of note, both antigens were discovered on the same CFA/I-encoding plasmid [which also encodes a copy of STh (26)] that was cured in the H10407-P strain, indicating that multiple virulence factors were eliminated with CFA/I in preparation of this isolate.

The first of these, EatA, is a member of the serine protease autotransporters of Enterobacteriaciae (SPATE) family. EatA (27), a close homologue of SepA, a major secreted protein discovered in Shigella flexneri (28), appears to function primarily to degrade MUC2 mucin (29) secreted by goblet cells of intestinal epithelia, potentially overcoming the mucin barrier to accelerate access of the bacteria to the epithelial surface, and promote successful delivery of both enterotoxins.

A second secreted molecule which appears to be exclusive to ETEC is encoded by the etpBAC locus of three plasmid-borne genes that result in production of a two-partner secretion system in which EtpB corresponds to an outer membrane transporter for EtpA, a secreted adhesin that is glycosylated by the EtpC glycosyltransferase (30). EtpA appears to act by serving as molecular bridge between the bacterial surface and its flagellar appendages (31) and GalNAc-containing host cell glycans including those present in mucin (32) and the human A blood group (33).

Both EtpA and the $110 \mathrm{kd}$ secreted EatA passenger domain are recognized during the course of natural and experimental controlled human infections. Both proteins are immunogenic in preclinical vaccine animal studies and antibodies against these proteins impact small intestinal colonization in mice (29, 34-37), as well as bacterial adhesion and toxin delivery in vitro. Other than LT and ST, these proteins appear to be the most highly conserved virulence proteins specific to the ETEC pathovar, and are widely distributed in a global collection of strains from diverse geographic sources (38-41). In a recent analysis of a birth cohort of young children in Bangladesh followed from birth to 24 months of age, both proteins were highly associated with symptomatic infection (42). Conversely, acquisition of antibodies to either protein was associated with asymptomatic intestinal colonization. The preponderance of evidence now suggests that these more recently discovered proteins play vital roles in the molecular pathogenesis of ETEC, although additional studies will be required to examine their role as protective antigens.

A third antigen, YghJ (SslE), is encoded on the chromosome of multiple ETEC isolates immediately upstream from the type II secretion system that is responsible for export of both LT and YghJ (43). YghJ degrades intestinal mucins to promote access of bacteria to intestinal epithelial cells promoting toxin delivery (44). YghJ is highly conserved, is not specific to the ETEC pathovar, and is found in extraintestinal E. coli (ExPEC) as 
well as some commensal isolates, including the well characterized Nissle 1917 strain. It appears to play important roles in biofilm formation and intestinal colonization in murine models of infection, where it has also been shown to be effective as a protective antigen $(45,46)$.

\section{IMPACT OF GENOMICS AND IMMUNOPROTEOMICS ON ETEC VACCINOLOGY}

E. coli in general exhibit remarkable genetic plasticity with an "open" pangenome, such that each sequenced genome results in the identification of approximately 300 unique genes (47), potentially posing a challenge to development of a broadly protective vaccine. The diversity of ETEC is further reflected in the multiple $\mathrm{O}$ and $\mathrm{H}$ serotypes that express one or both enterotoxins suggesting that the plasmids encoding ETEC virulence factors are widely distributed in an otherwise diverse population of E. coli $(48,49)$. However, the availability of hundreds of ETEC genomes has recently permitted an unprecedented ability to assess antigenic conservation across a wide variety of isolates from disparate sources (50). In addition, databases that incorporate hundreds of genomes are now easily accessible through online data visualization tools to facilitate comparison of bacterial genomes and associated metadata (51). Data for more than 1000 sequenced ETEC genomes and their associated metadata appear here: https://microreact.org/project/ 2ZZzaHzeXbMEw9U2MAk7pK?tt=cr. Despite the extraordinary heterogeneity of ETEC genomes, a number of recent studies now suggest that successful lineages of ETEC share common virulence plasmids $(41,50,52)$, and a finite number of potential surface-expressed antigenic targets restricted to ETEC (53) that might be exploited to complement canonical approaches to vaccine development $(38,40,54)$.

Access to multiple genomes has also accelerated the advancement of ETEC proteome microarrays that include hundreds of antigens that can be screened with convalescent sera or following vaccination. Exploitation of these microarrays can further inform vaccine antigen selection by highlighting candidate immunogens. Arrays developed recently used reverse vaccinology pipelines to identify candidate surface antigens common to large numbers of ETEC but absent from sequenced commensal or laboratory isolates of E. coli. Notably, even with an open-aperture approach that encompasses many potential as well as known surface protein use of these arrays has revealed that the ETEC-specific immunoproteome is restricted to a finite number of surface antigens with a surprisingly small number of molecules that are recognized during the course of infection $(42,55,56)$. As prior infection with ETEC is associated with protection, these studies can likewise highlight potential protective antigens. Indeed, using these platforms to compare responses to vaccination with the live-attenuated ETEC ACE527 vaccine to those following infection with ETEC revealed responses unique to infection that were absent following vaccination (56).

\section{CORRELATES OF PROTECTION FOLLOWING ETEC INFECTION AND VACCINATION}

\subsection{Immunity Following Natural and Experimental Human Infection}

The incidence of ETEC diarrhea among children in LMICs peaks before 2 years of age (2) with subsequent declines thereafter such that the bulk of symptomatic infections occur in children under the age of 5 years (57). At present there is no clear mechanistic correlate of protection following ETEC infection (58-60), and studies vary with regard to the overall impact of prior infection $(61,62)$ or infection with strains of particular toxin/CF profiles and protection against symptomatic diarrhea with strains of the same profile $(59,63,64)$. Notably, homologous re-challenge with the same strain following controlled human infection typically results in robust protection, while rechallenge with a different strain has not (65), suggesting that homotypic immunity develops following an initial ETEC infection, while heterotypic immunity may be limited.

\subsubsection{The Importance of Memory}

Although secretory IgA directed at ETEC virulence factors is widely thought to be important, sustained protection likely requires development of effective $B$ cell memory responses $(66,67)$. Studies in human volunteers further also suggest that a subset of CD 4 cells, $\alpha 4 \beta 7$-expressing circulating antigenspecific $\mathrm{T}$ follicular helper cells home to gut associated lymphoid tissues to interact with B cells to elicit B memory responses (68). Here, higher levels of $\alpha 4 \beta 7$ expression were associated increased antigen-specific IgA B cell memory and with lower stool volumes in volunteers following challenge.

Likewise, in naturally infected Bangladeshi adults ETEC infections were associated with specific memory B cell responses to colonization factors and heat labile toxin (69). These responses were associated with development high avidity antibodies to these antigens suggesting that they may participate in neutralizing anamnestic responses.

Similar memory responses can be elicited by vaccination with the oral whole-cell killed ETEC vaccine ETVAX. Studies in human volunteers demonstrated that re-administration of the ETVAX more than one year later elicited substantial increases in antibody lymphocyte supernatant (ALS) IgA directed at CFs and LT, evidence that the vaccine induced sustained mucosal immunological memory (70). As seen with infection with wild type ETEC, vaccination with ETVAX also led to increases in circulating activated $\mathrm{T}$ follicular helper cells (cTfh) that correlated with vaccine-specific ALS IgA production (71), suggesting that measuring these cells could serve as an effective markers of vaccine "take" and prediction of long term mucosal memory responses.

\subsubsection{The Impact of Repeated Natural Infection in Endemic Regions}

In contrast to travelers, children in LMICs are repeatedly exposed to ETEC, potentially providing a strong and repetitive 
antigenic stimulus following initial exposure. Repeated symptomatic infections in this population (2) make it clear that natural infection does not necessarily induce sterilizing immunity, although re-exposures in highly endemic environments may elicit anamnestic responses that sustain protection following vaccination

\subsubsection{Protection Following Vaccination}

As data from cohort studies in which children were followed from birth to the age of 24 months demonstrate that symptomatic re-infection with ETEC strains expressing the same colonization factor (CF) is not common (2), most vaccines have attempted to elicit vaccine responses to CFs (7274) and to correlate mucosal responses to the targeted antigens to protection. While precise mechanistic correlates of protection are not evident from studies to date, comparison of immune profiles generated during controlled human infection model (CHIM) studies (55) to vaccination can be instructive. Proteome microarray profiling of immune responses to the live attenuated ETEC vaccine ACE527 with or without the mucosal adjuvant dmLT (LT R192G/L211A) revealed that both ACE527 (protective efficacy, PE 27\%) (75) and ACE527 + dmLT (PE 66\%) (76) elicited similar responses to canonical antigens targeted in the vaccine (CS1,CS2, CS3, CS5, CS6, CFA/I, LT-B), yet failed to elicit responses to other antigens, including EtpA and flagellin, recognized following challenge with wild type ETEC, which affords almost complete protection on homologous re-challenge $(55,56)$. Although clinical trials of ETEC vaccines have attempted to correlate responses to colonization factors and LT with protection, the studies of ACE527 highlight difficulties inherent in attempting to mimic the complex and multifaceted responses to infection that ultimately result in protection.

\subsubsection{Cytokine Responses to ETEC Infection}

Controlled human infection model (CHIM) studies have provided some insight into the nature of cytokine responses related to acute ETEC infections. In volunteer studies, serum levels of IL-17 and interferon $\gamma$ increase significantly in subjects with moderate-severe diarrheal illness (77). In the gut IL-17 is produced by a variety of cell types, including Paneth cells which reside at the base of the crypts in the small intestine. Here, IL-17 plays a critical role in maintaining mucosal integrity, in promoting release of antimicrobial compounds including defensins, and in stimulating pro-inflammatory cytokines (78). Importantly, LT has been shown to induce production of IL-1ß and IL-23 secretion by dendritic cells which then promote antigen-specific IL-17 and interferon- $\gamma$ production in CD4+ T cells. A mixed Th1/Th2 response accompanied by robust Th17 induction and migration of gut homing $\alpha 4 \beta 7$-expressing lymphocytes account for the potent adjuvant activity of LT (79). The B-cell help provided by IL-17 promotes germinal center formation (80) and elicits production of secretory IgA at mucosal surfaces to neutralize the pathogen and/or its toxins. Incorporation of dmLT or other adjuvants that can recapitulate responses to the native toxin will likely be important in achieving robust and sustained mucosal protection against ETEC (81).

\section{VACCINE PIPELINE}

A number of candidate vaccines ETEC based on the canonical virulence paradigm have progressed to clinical investigation. Each of these is designed to target multiple CF/CS antigens \pm LT (Table 1).

\subsection{Cellular Vaccines Based on the Classical ETEC Pathogenesis Paradigm}

All ETEC vaccines to enter clinical testing thus far are centered on the classical paradigm for ETEC virulence and attempt to interrupt intestinal colonization by engendering responses to select colonization factor (CF or CS) antigens and LT. These have taken the form of mixtures of whole cell killed $(91,92)$, or liveattenuated $(75,76,93)$ ETEC strains engineered to express one or more $\mathrm{CF} / \mathrm{CS}$ antigens. Most studies indicate that multiple CF/CS antigens would be needed to afford broad coverage in an ETEC vaccine although when combined they could cover $\sim 3 / 4$ of all isolates expressing the most common colonization factors (94), and $1 / 4$ of more of the strains lack one of the established, previously characterized CF/CS antigens (54, 95-98).

ETVAX is a whole-cell inactivated mixture of ETEC strains developed at the University of Gothenburg to target major CFs/ CS antigens (CFA/I, CS3, CS5, CS6) and includes a novel LT toxoid LCTBA in addition to the double mutant LT adjuvant, dmLT. In a randomized placebo-controlled double-blind phase 1/II dose escalation study in Bangladeshi children 6-59 months of age, the vaccine was generally safe and the majority of vaccine recipients mounted significant mucosal IgA responses against each of these antigens (73), suggesting that it could effectively prevent infection in a major target population.

ETVAX has also been studied (phase 2b) in Finnish travelers to Benin, West Africa where there was an appreciable attack rate of ETEC diarrhea, and ETEC featured prominently in ( 75\%) cases of severe diarrhea ( $>16$ loose stools/24 hours). In vaccine responders the protective efficacy was $\sim 52 \%$, and far fewer vaccine recipients required antimicrobial treatment to mitigate severe illness suggesting that the vaccine was generally effective (20, 99).

ACE527 is an orally administered vaccine mixture of three live-attenuated strains engineered to express CFA/I and CS1CS3, CS5, and CS6 in addition to the B subunit of heat-labile toxin (LT-B) $(93,100)$. Controlled human infection model studies of ACE527 demonstrated that when given alone, it failed to significantly protect against more severe forms of diarrhea upon challenge with ETEC H10407, although it did reduce colonization (75). In contrast, addition of dmLT to ACE527 resulted in marked decrease in shedding of the H10407 challenge strain commensurate with a significant increase in protective efficacy (66\%) against H10407 challenge (76). Subsequent protein microarray studies undertaken to examine differences in immune responses to wild type H10407 relative to ACE527 and ACE527 adjuvanted with dmLT revealed that the vaccine with or without dmLT engendered comparable immune responses to target canonical antigens, but that immune responses to potentially protective novel antigens including the EtpA adhesin, the YghJ metalloprotease, and flagellin were 
TABLE 1 | Current landscape of ETEC vaccine development.

\begin{tabular}{|c|c|c|c|c|c|c|c|}
\hline \multirow[t]{2}{*}{ Vaccine candidate } & \multirow[t]{2}{*}{ Vaccine type/description } & \multirow[t]{2}{*}{ Target antigens } & \multicolumn{4}{|c|}{ Clinical phase } & \multirow[t]{2}{*}{ Reference(s) } \\
\hline & & & pre & 1 & 2 & 3 & \\
\hline \multicolumn{8}{|l|}{ Whole cell-bacteria } \\
\hline$E T V A X \pm d m L T$ & whole-cell inactivated, recombinant LTCBA & CFA/I, CS3,5,6; LT & & & a & & (73) \\
\hline ACE527 \pm dmLT & live-attenuated, recombinant LT-B & CFA/I, CS1-3,5,6, LT & & & b & & $(75)$ \\
\hline Shigella-ETEC multivalent & live-attenuated Shigella-ETEC heterologous expression & CFA/I, CS1-6, 14; LThA2B & & & & & $(82)$ \\
\hline \multicolumn{8}{|l|}{ Recombinant subunits } \\
\hline dmLT LT(R192G/L211A) & recombinant antigen/adjuvant & LT & & $c-f$ & & & $(73,75,83,84)$ \\
\hline LT-ST fusion (ENTVAC) & recombinant toxoid fusion STa(N12S) ${ }^{3}-\mathrm{LT}(\mathrm{R} 192 \mathrm{G} / \mathrm{L} 211 \mathrm{~A})$ & LT/ST & & & & & $(85,86)$ \\
\hline \multirow[t]{3}{*}{ fimbrial tip adhesins } & recombinant dscCfaE + LT(R192G) & CFA/I, LT & & g & h & & $(87)$ \\
\hline & recombinant cssBA $\pm d m L T$ & CS6, LT & & $\mathbf{i}$ & & & (88) \\
\hline & chimeric recombinant dsc14CfaE-sCTA2/LTB5 & CFA/I, LT & & j & & & (89) \\
\hline MEFA & multi-epitope fusion antigen $+\mathrm{dmL} T$ & CFA/I, CS1-6, LT & & & & & $(90)$ \\
\hline EtpA & recombinant adhesin & EtpA & & & & & $(36,37)$ \\
\hline EatA & recombinant mucin-degrading protease & EatA & & & & & (29) \\
\hline YghJ(SslE) & recombinant metalloprotease & YghJ(SslE) & & & & & $(45)$ \\
\hline
\end{tabular}

Clinical trials identifier.

a NCT02531802, NCT03729219.

${ }^{\boldsymbol{b}}$ NCT01739231.

cNCT02052934 (sl dmLT).

d NCT01147445 oral dose escalation.

eNCT02531685.

${ }^{f}$ NCT03548064 oral, sublingual, intradermal in Bangladesh.

${ }^{\text {gNCT01382095. }}$

${ }^{n}$ NCT01922856.

iNCT03404674cssBA.

${ }^{j}$ NCT01644565.

markedly diminished or absent relative to wild type H10407 challenge (56). While ACE527 is not currently under further development, these studies importantly emphasized responses following ETEC infection that are distinct from vaccination and which may be critical for efficient protection.

A second live-attenuated platform under development by the Center for Vaccine Development (CVD) at the University of Maryland relies on heterologous presentation of ETEC antigens in Shigella. The prototype Shigella strain CVD 1208S, derived from Shigella flexneri 2a, contains attenuating mutations in $g u a B A$, and two enterotoxin genes sen and set, and was found to be safe and immunogenic in phase 1 clinical studies (NCT01531530) (101). This strain has been engineered to express CFA/I and the A2 and B subunits of LT (LThA2B) from the chromosome, and tested in murine models where it was shown to mitigate weight loss and diarrhea following challenge with either CFA/I-expressing ETEC or Shigella flexneri 2A (102). CVD is currently engineering a multivalent Shigella-ETEC vaccine that incorporates $\Delta g u a B A \Delta s e n \Delta s e t$ mutations in the most prevalent serotypes of Shigella flexneri (2a, 3a, 6, 1b) found in the Global Enteric Multicenter Study (GEMS) (3), in addition to Shigella sonnei and dysenteriae, with each expressing heterologous antigens to cover CS1-3, CS5,CS6, CS14 and CFA/I with the LThA2B toxoid (82).

\subsection{Acellular Subunit Candidates 5.2.1 Development of Toxoids}

Successful delivery of ETEC ST and LT enterotoxins is the sine qua non of ETEC molecular pathogenesis. Theoretically, interruption of any step which mitigates toxin binding to the respective epithelial cell surface receptors, including toxoids, could be exploited in development of a vaccine. Indeed, toxoids form the basis for a number of licensed subunit childhood subunit vaccines against toxigenic bacterial pathogens including diphtheria, and pertussis, suggesting that this is a viable approach. Similarly, there is ample reason to hope that toxoids against both LT and ST could form the basis of a broadly protective vaccine strategy.

LT is inherently immunogenic, and most studies have demonstrated that there is a robust response to the toxin following infection. Studies of the oral whole-cell cholera vaccine combined with cholera toxin (CT) B subunit (Dukoral) in travelers demonstrated significant cross protection afforded by the antigenically similar CT B subunit against LT+ ETEC (103), providing enthusiasm for targeting LT. Subsequent studies of LT transdermal delivery via patch demonstrated efficacy in mitigating the severity and duration of diarrheal illness following controlled clinical challenge with a strain that produces both LT and ST (104). Likewise transdermal delivery of LT demonstrated some efficacy in lessening the severity of traveler's diarrhea caused by strains that produced LT, and overall in preventing more severe forms of diarrheal illness of all causes (105). In a larger phase III study in travelers, protective efficacy $(\sim 61 \%)$ was limited to strains which produced LT only (106). Altogether, these results suggest that direct targeting of LT using some version of LT toxoid would at least provide some protection against LT-producing ETEC, although other antigens would almost certainly be required for broad protection (107).

The double mutant LT(R192G/L211A) form of LT (dmLT) (Figure 1A) retains ADP-ribosylating activity and the 
immunogenicity of the parent molecule and exhibits potent mucosal adjuvant activity for co-administered antigens, making it an ideal component of ETEC vaccines (81). dmLT has proven to be safe when administered either orally (83), via sublingual route (84), or parenterally. Doses of 50-100 $\mu$ g are well-tolerated orally, and elicit significant mucosal antibody responses against LT (83). Encouraging data from both preclinical and clinical studies have accelerated inclusion of $\mathrm{dmLT}$ in candidate vaccines, and the significantly enhanced protective efficacy observed on administration of dmLT with the live-attenuated ACE527 ETEC vaccine highlights its utility as a potent mucosal adjuvant (76).

Until recently, heat-stable toxins were not considered to be viable candidates for toxoid development for a number of reasons. First both versions of heat-stable toxin found in strains that infect humans, ST-H and ST-P, are small (19 and 18 amino acids, respectively) poorly immunogenic peptides (Figure 1B). In addition, both molecules bind to quanylate cyclase $\mathrm{C}$ (GC-C) on the surface of enterocytes where they act as molecular mimics for the peptides guanylin and uroguanylin which normally regulate fluid homeostasis in the human gastrointestinal tract. Indeed, the FDA-approved drug linaclotide, a close relative of ST-H, differs from the core region of the toxin by only a single amino acid (112), and is designed to exploit this mimicry in the treatment of chronic constipation and irritable bowel syndrome. Therefore, there was a legitimate concern that engendering antibodies against ST peptides would cross react with the endogenous peptide ligands and interfere with fluid homeostasis. This concern was substantiated in studies in which antibodies raised against STh or STp conjugates cross-reacted with both endogenous peptides $(113,114)$.

Detailed screening of a library of STh amino acid substitutions has however delineated mutations within STh such as A14T
(Figure 1C) that exhibit substantially reduced toxicity and induce ST neutralizing antibodies $(113,115-117)$ which do not cross react appreciably with either of the endogenous GC-C agonist peptides. Similarly, presentation of STh (A14T) on the surface of self-assembling nanoparticles elicited robust ST neutralizing antibodies without substantial cross reaction with either guanylin or uroguanylin (118).

The promise of toxoids to afford protection is further highlighted by studies in which copies of mutant ST were genetically fused to LTA (R192G/L211A) and single copy of LTB. Immunization of pregnant pigs with this construct adjuvanted with dmLT yielded substantial protection against diarrhea in suckling pigs following challenge with an ST-producing strain (85).

In summary, the current evidence suggests that both toxins could be effectively targeted to engender neutralizing antibody responses, interrupting a critical step in ETEC molecular pathogenesis. These collective advancements in development of toxoids for both ST and LT provide strong impetus for pursuing their development as protective immunogens.

\subsubsection{Development of CF and CS Subunit Vaccines}

Elegant structural studies of CF and CS biogenesis have informed the development of protective antigenic subunits (119). The most advanced of these, based on the donor strand complemented (dsc) tip adhesin structure of CFA/I, dscCfaE, was used previously to generate bovine immune colostral IgG antibodies that afforded robust passive protection against subsequent challenge with H10407 (120). This tip adhesin complex was subsequently tested for immunogenicity when delivered transcutaneously with mutant LT (R192G) via patch in human volunteers (87). Although these studies indicate that additional effort will be required for optimal delivery of the fimbrial tip adhesin complex (121), recent studies of
A

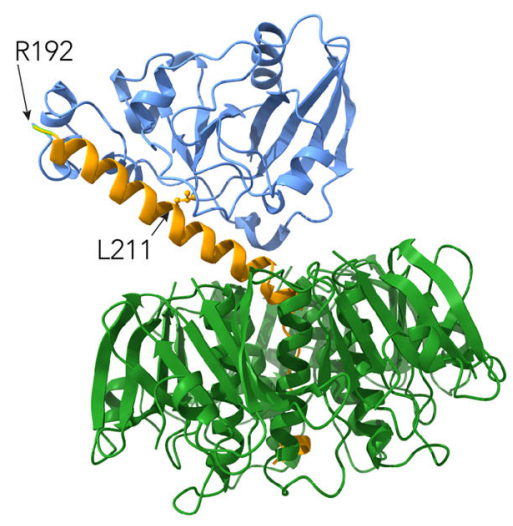

B

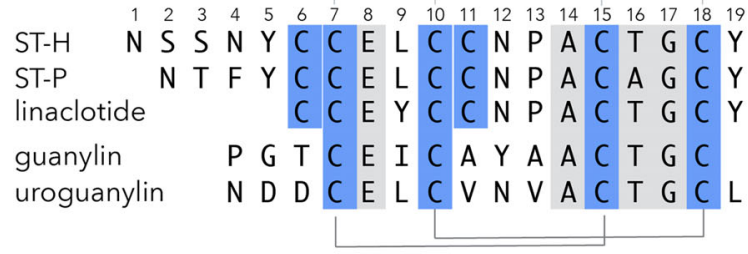

C

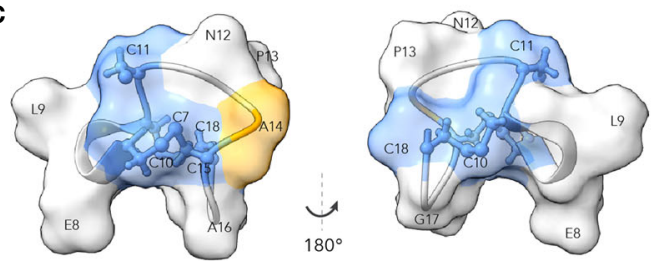

FIGURE 1 | Molecular basis of toxoid development for ETEC. (A) ETEC heat-labile toxin based on Protein Data Bank (PDB) structure 1LTS (108), with the A1 subunit in blue, A2 in yellow, and the pentameric B subunit in green. Location of R192G and L211A mutations in dmLT are indicated by the arrows. (B) Sequence alignment of ETEC heat-stable toxins STh and STp, linaclotide, and native peptides guanylin and uroguanylin. Disulfide bonds are indicated above and below the alignment. Numbers refer to the amino acid sequence of STh. (C) Surface structural model of core ST toxin domain of (amino acids C6-C18) based on STp PDB structure 1ETN (109). Regions in blue depict location of cysteines. Location of candidate toxoid mutation at A14, part of the putative GC-C receptor binding site formed by N12-A14 (110), is shown in yellow. Molecules shown were displayed using ChimeraX v1.1.1 (111). 
dscCfaE genetically fused in a chimeric complex with the A2 subunit of cholera toxin and the B subunit of LT (dscCfaEsCTA2/LTB) resulted in substantial protection in an Aotus nancymaae model of infection, following intradermal vaccination with mLT(R192G) (89).

Similar preclinical studies of CS6, an antigen present in $\sim 1 / 5$ of ETEC strains have been ongoing. CS6 is comprised of two major subunits CssA and CssB, and early results with recombinant CssBA of Css $A B$ heterodimers suggest that it is possible to generate neutralizing antibodies to CS6 (88). A phase 1 safety and immunogenicity study of CssBA + dmLT administered IM has been completed although data on protective efficacy is currently pending. Interestingly, however administration of hyperimmune bovine IgG against recombinant CS6 did not afford significant protection against moderate to severe diarrhea upon challenge with a well-characterized CS6-producing strain B7A (122).

A potential pitfall inherent in the adhesin subunit approach is that more than 25 distinct CF/CS antigens have been identified to date, complicating the path to a broadly protective multivalent vaccine. Although it remains to be seen to what degree broadbased protection can be achieved with a limited number of antigens, there is significant cross immunologic reactivity of class 5 fimbrial tip adhesins $(72,123,124)$.

Development of a multiepitope fusion antigen (MEFA) that incorporates epitopes from multiple antigens represents an attempt to overcome valency thresholds that would be required to achieve broad protection. The most recent iteration of the MEFA platform incorporates ST-LT fusion toxoid molecules combining both antiadhesin and toxoid approaches (125). Notably, this latest iteration of MEFA was protective in a porcine challenge model suggesting that the toxoid induces effective neutralizing antibodies.

\subsubsection{Non-Canonical Antigen Subunits}

A potential complementary approach to achieving broad coverage against a diverse population of ETEC would be to target other surface-expressed "non-canonical" antigens (i.e., other than the CF/ CS antigens and toxins) that could be targeted to mitigate effective toxin delivery. Expanded genomic, immunoproteomic, molecular pathogenesis, as well as clinical investigation efforts have highlighted several antigens including the EtpA adhesin, the EatA mucinase, and the YghJ (SslE) metalloprotease that have not traditionally been targets for ETEC vaccines.

The relative conservation of each of these antigens within the ETEC pathovar, their immunogenicity during natural (42) and experimental human infections (55), and molecular pathogenesis studies all suggest that these antigens, which have not been targets of ETEC vaccines to date, play important roles in ETEC infection. Nevertheless, additional effort will be needed to accelerate their implementation in future iterations of ETEC vaccines.

Interestingly, although these antigens have demonstrated some efficacy in animal models of infection $(29,37)$, it is not currently known whether any of these molecules (all of which are secreted from ETEC) would have been retained in whole cell vaccines such at ETVAX which have reached advanced stages of development. Notably, genes responsible for secretion of EtpA were inadvertently deleted in construction of ACE527, likely in engineering of the parent strains to remove toxin genes $(93,100)$, and each of the resulting vaccine strains were immotile potentially impacting efficacy of relative to challenge with wild type bacteria (56).

\section{CHALLENGES FOR DEVELOPMENT}

The Department of Immunization, Vaccines and Biologicals of the WHO has published "Preferred Product Characteristics" to guide development of ETEC vaccines designed for target populations in LMICs (126). In this context, a vaccine that is affordable and that significantly reduces mortality and morbidity associated with moderate to severe diarrhea among children under the age of 5 years in LMICs is the primary aspirational goal.

A number of challenges present barriers to achieving this goal. These include lack of broadly applicable testing to assess disease burden, particularly in remote areas where incidence may be quite high; lack of clear correlates of protection; as well as animal models that do not completely recapitulate human disease necessitating a reliance on controlled human infection models (CHIM) (127) testing for accurate prioritization and down-selection of vaccine candidates.

Affordability will undoubtably play an essential role in guiding global vaccine value assessments for vaccine prioritization. The complex nature of ETEC and the underlying genetic plasticity of $E$. coli could impede progress toward development toward a broadly protective vaccine suitable for deployment to LMICs.

The extraordinary CF/CS structural and antigenic diversity poses a substantial challenge to vaccine approaches exclusively centered on these antigens. Antibodies that recognize several closely related CF adhesin molecules may have functional activity restricted to a single antigen (123), highlighting difficulties inherent to practical development of a vaccine solely based on CFs.

Additional effort will therefore be needed to define complementary strategies. Advanced development of hybrid toxoids that protect against ST and LT, a more complete understanding of ETEC molecular pathogenesis that intensifies focus on more highly conserved virulence features, particularly those which play a role in development of more severe forms of illness (42), and elucidation of the cellular events underlying ETECs relationship to sequelae including stunting and malnutrition may be needed to inform the construction of a truly affordable and broadly protective vaccine.

\section{AUTHOR CONTRIBUTIONS}

The author confirms being the sole contributor of this work and has approved it for publication.

\section{FUNDING}

This work was supported in part with funding from the National Institutes of Allergy and Infectious Diseases (NIAID) of the National Institutes of Health (NIH) by grants R01AI089894, and R01AI126887; the Department of Veterans Affairs I01 BX004825-01. 


\section{REFERENCES}

1. Khalil IA, Troeger C, Blacker BF, Rao PC, Brown A, Atherly DE, et al. Morbidity and Mortality Due to Shigella and Enterotoxigenic Escherichia Coli Diarrhoea: The Global Burden of Disease Study 1990-2016. Lancet Infect Dis (2018) 18(11):1229-40. doi: 10.1016/S1473-3099(18)30475-4

2. Qadri F, Saha A, Ahmed T, Al Tarique A, Begum YA, Svennerholm AM. Disease Burden Due to Enterotoxigenic Escherichia Coli in the First 2 Years of Life in an Urban Community in Bangladesh. Infect Immun (2007) 75 (8):3961-8. doi: 10.1128/IAI.00459-07

3. Kotloff KL, Nataro JP, Blackwelder WC, Nasrin D, Farag TH, Panchalingam $\mathrm{S}$, et al. Burden and Aetiology of Diarrhoeal Disease in Infants and Young Children in Developing Countries (The Global Enteric Multicenter Study, GEMS): A Prospective, Case-Control Study. Lancet (2013) 382(9888):20922. doi: 10.1016/S0140-6736(13)60844-2

4. Bagamian KH, Anderson JD IV, Muhib F, Cumming O, Laytner LA, Wierzba TF, et al. Heterogeneity in Enterotoxigenic Escherichia Coli and Shigella Infections in Children Under 5 Years of Age From 11 African Countries: A Subnational Approach Quantifying Risk, Mortality, Morbidity, and Stunting. Lancet Glob Health (2020) 8(1):e101-12. doi: 10.1016/S2214109X(19)30456-5

5. Anderson JDT, Bagamian KH, Muhib F, Amaya MP, Laytner LA, Wierzba T, et al. Burden of Enterotoxigenic Escherichia Coli and Shigella Non-Fatal Diarrhoeal Infections in 79 Low-Income and Lower Middle-Income Countries: A Modelling Analysis. Lancet Glob Health (2019) 7(3):e321-30. doi: 10.1016/S2214-109X(18)30483-2

6. Dalton CB, Mintz ED, Wells JG, Bopp CA, Tauxe RV. Outbreaks of Enterotoxigenic Escherichia Coli Infection in American Adults: A Clinical and Epidemiologic Profile. Epidemiol Infect (1999) 123(1):9-16. doi: 10.1017/S0950268899002526

7. Lamberti LM, Bourgeois AL, Fischer Walker CL, Black RE, Sack D. Estimating Diarrheal Illness and Deaths Attributable to Shigellae and Enterotoxigenic Escherichia Coli Among Older Children, Adolescents, and Adults in South Asia and Africa. PloS Negl Trop Dis (2014) 8(2):e2705. doi: 10.1371/journal.pntd.0002705

8. Medus C, Besser JM, Juni BA, Koziol B, Lappi V, Smith KE, et al. Long-Term Sentinel Surveillance for Enterotoxigenic Escherichia Coli and Non-O157 Shiga Toxin-Producing E. Coli in Minnesota. Open Forum Infect Dis (2016) 3(1):ofw003. doi: 10.1093/ofid/ofw003

9. Naimi TS, Wicklund JH, Olsen SJ, Krause G, Wells JG, Bartkus JM, et al. Concurrent Outbreaks of Shigella Sonnei and Enterotoxigenic Escherichia Coli Infections Associated With Parsley: Implications for Surveillance and Control of Foodborne Illness. J Food Prot (2003) 66(4):535-41. doi: 10.4315/ 0362-028X-66.4.535

10. Beatty ME, Adcock PM, Smith SW, Quinlan K, Kamimoto LA, Rowe SY, et al. Epidemic Diarrhea Due to Enterotoxigenic Escherichia Coli. Clin Infect Dis (2006) 42(3):329-34. doi: 10.1086/499246

11. Beatty ME, Bopp CA, Wells JG, Greene KD, Puhr ND, Mintz ED. Enterotoxin-Producing Escherichia Coli O169:H41, United States. Emerg Infect Dis (2004) 10(3):518-21. doi: 10.3201/eid1003.030268

12. Devasia RA, Jones TF, Ward J, Stafford L, Hardin H, Bopp C, et al. Endemically Acquired Foodborne Outbreak of Enterotoxin-Producing Escherichia Coli Serotype O169:H41. Am J Med (2006) 119(2):168.e7-10. doi: 10.1016/j.amjmed.2005.07.063

13. Roels TH, Proctor ME, Robinson LC, Hulbert K, Bopp CA, Davis JP. Clinical Features of Infections Due to Escherichia Coli Producing Heat-Stable Toxin During an Outbreak in Wisconsin: A Rarely Suspected Cause of Diarrhea in the United States. Clin Infect Dis (1998) 26(4):898-902. doi: 10.1086/513923

14. Jain S, Chen L, Dechet A, Hertz AT, Brus DL, Hanley K, et al. An Outbreak of Enterotoxigenic Escherichia Coli Associated With Sushi Restaurants in Nevada, 2004. Clin Infect Dis (2008) 47(1):1-7. doi: 10.1086/588666

15. Yoder JS, Cesario S, Plotkin V, Ma X, Kelly-Shannon K, Dworkin MS. Outbreak of Enterotoxigenic Escherichia Coli Infection With an Unusually Long Duration of Illness. Clin Infect Dis (2006) 42(11):1513-7. doi: 10.1086/ 503842

16. Centers for Disease Control and Prevention (CDC). Foodborne Outbreaks of Enterotoxigenic Escherichia Coli-Rhode Island and New Hampshire, 1993. MMWR Morb Mortal Wkly Rep (1994) 43(5):81, 87-9.
17. Reiner RC Jr, Graetz N, Casey DC, Troeger C, Garcia GM, Mosser JF, et al Variation in Childhood Diarrheal Morbidity and Mortality in Africa, 20002015. N Engl J Med (2018) 379(12):1128-38. doi: 10.1056/NEJMoa1716766

18. Anderson JDT, Muhib F, Rheingans R, Wierzba T. Heterogeneity in Potential Impact and Cost-Effectiveness of ETEC and Shigella Vaccination in Four Sub-Saharan African Countries. Vaccine X (2019) 3:100043. doi: 10.1016/j.jvacx.2019.100043

19. Hosangadi D, Smith PG, Giersing BK. Considerations for Using ETEC and Shigella Disease Burden Estimates to Guide Vaccine Development Strategy. Vaccine (2019) 37(50):7372-80. doi: 10.1016/j.vaccine.2017.09.083

20. WHO. WHO Product Development for Vaccines Advisory Committee (PDVAC). Update on Development of Enterotoxigenic E. Coli (ETEC) Vaccines. (2020). Available at: https://cdn.who.int/media/docs/default-source/immunization/ pdvac/2020/pdvac_etec-vaccines_18-june_presentations_reduced.pdf?sfvrsn= 930ceb78_8 (Accessed 2021 05/10/2021). 18 June 2020.

21. Nalin DR, Bhattacharjee AK, Richardson SH. Cholera-Like Toxic Effect of Culture Filtrates of Escherichia Coli. J Infect Dis (1974) 130(6):595-607. doi: 10.1093/infdis/130.6.595

22. Sack RB, Gorbach SL, Banwell JG, Jacobs B, Chatterjee BD, Mitra RC. Enterotoxigenic Escherichia Coli Isolated From Patients With Severe Cholera-Like Disease. J Infect Dis (1971) 123(4):378-85. doi: 10.1093/ infdis/123.4.378

23. Carpenter CC, Barua D, Wallace CK, Sack RB, Mitra PP, Werner AS, et al. Clinical and Physiological Observations During an Epidemic Outbreak of Non-Vibrio Cholera-Like Disease in Calcutta. Bull World Health Organ (1965) 33(5):665-71.

24. Evans DG, Silver RP, Evans DJ Jr, Chase DG, Gorbach SL. PlasmidControlled Colonization Factor Associated With Virulence in Escherichia Coli Enterotoxigenic for Humans. Infect Immun (1975) 12(3):656-67. doi: 10.1128/iai.12.3.656-667.1975

25. Satterwhite TK, Evans DG, DuPont HL, Evans DJ Jr. Role of Escherichia Coli Colonisation Factor Antigen in Acute Diarrhoea. Lancet (1978) 2 (8082):181-4. doi: 10.1016/S0140-6736(78)91921-9

26. Crossman LC, Chaudhuri RR, Beatson SA, Wells TJ, Desvaux M, Cunningham AF, et al. A Commensal Gone Bad: Complete Genome Sequence of the Prototypical Enterotoxigenic Escherichia Coli Strain H10407. J Bacteriology (2010) 192(21):5822-31. doi: 10.1128/JB.00710-10

27. Patel SK, Dotson J, Allen KP, Fleckenstein JM. Identification and Molecular Characterization of Eata, An Autotransporter Protein of Enterotoxigenic Escherichia Coli. Infect Immun (2004) 72(3):1786-94. doi: 10.1128/ IAI.72.3.1786-1794.2004

28. Benjelloun-Touimi Z, Sansonetti PJ, Parsot C. Sepa, the Major Extracellular Protein of Shigella Flexneri: Autonomous Secretion and Involvement in Tissue Invasion. Mol Microbiol (1995) 17(1):123-35. doi: 10.1111/j.13652958.1995.mmi_17010123.x

29. Kumar P, Luo Q, Vickers TJ, Sheikh A, Lewis WG, Fleckenstein JM. Eata, an Immunogenic Protective Antigen of Enterotoxigenic Escherichia Coli, Degrades Intestinal Mucin. Infect Immun (2014) 82(2):500-8. doi: 10.1128/IAI.01078-13

30. Fleckenstein JM, Roy K, Fischer JF, Burkitt M. Identification of a TwoPartner Secretion Locus of Enterotoxigenic Escherichia Coli. Infect Immun (2006) 74(4):2245-58. doi: 10.1128/IAI.74.4.2245-2258.2006

31. Roy K, Hilliard GM, Hamilton DJ, Luo J, Ostmann MM, Fleckenstein JM. Enterotoxigenic Escherichia Coli Etpa Mediates Adhesion Between Flagella and Host Cells. Nature (2009) 457(7229):594-8. doi: 10.1038/ nature 07568

32. Kumar P, Kuhlmann FM, Bhullar K, Yang H, Vallance BA, Xia L, et al. Dynamic Interactions of a Conserved Enterotoxigenic Escherichia Coli Adhesin With Intestinal Mucins Govern Epithelium Engagement and Toxin Delivery. Infect Immun (2016) 84(12):3608-17. doi: 10.1128/ IAI.00692-16

33. Kumar P, Kuhlmann FM, Chakraborty S, Bourgeois AL, Foulke-Abel J, Tumala B, et al. Enterotoxigenic Escherichia Coli-Blood Group a Interactions Intensify Diarrheal Severity. J Clin Invest (2018) 128(8):3298-311. doi: 10.1172/JCI97659

34. Roy K, Hamilton D, Allen KP, Randolph MP, Fleckenstein JM. The Etpa Exoprotein of Enterotoxigenic Escherichia Coli Promotes Intestinal Colonization and Is a Protective Antigen in an Experimental Model of 
Murine Infection. Infect Immun (2008) 76(5):2106-12. doi: 10.1128/ IAI.01304-07

35. Roy K, Hamilton D, Ostmann MM, Fleckenstein JM. Vaccination With Etpa Glycoprotein or Flagellin Protects Against Colonization With Enterotoxigenic Escherichia Coli in a Murine Model. Vaccine (2009) 27 (34):4601-8. doi: 10.1016/j.vaccine.2009.05.076

36. Roy K, Hamilton DJ, Fleckenstein JM. Cooperative Role of Antibodies Against Heat-Labile Toxin and the Etpa Adhesin in Preventing Toxin Delivery and Intestinal Colonization by Enterotoxigenic Escherichia Coli. Clin Vaccine Immunol (2012) 19(10):1603-8. doi: 10.1128/CVI.00351-12

37. Luo Q, Vickers TJ, Fleckenstein JM. Immunogenicity and Protective Efficacy Against Enterotoxigenic Escherichia Coli Colonization Following Intradermal, Sublingual, or Oral Vaccination With Etpa Adhesin. Clin Vaccine Immunol (2016) 23(7):628-37. doi: 10.1128/CVI.00248-16

38. Kuhlmann FM, Martin J, Hazen TH, Vickers TJ, Pashos M, Okhuysen PC, et al. Conservation and Global Distribution of Non-Canonical Antigens in Enterotoxigenic Escherichia Coli. PloS Negl Trop Dis (2019) 13(11): e0007825. doi: 10.1371/journal.pntd.0007825

39. Rasko DA, Del Canto F, Luo Q, Fleckenstein JM, Vidal R, Hazen TH. Comparative Genomic Analysis and Molecular Examination of the Diversity of Enterotoxigenic Escherichia Coli Isolates From Chile. PloS Negl Trop Dis (2019) 13(11):e0007828. doi: 10.1371/journal.pntd.0007828

40. Luo Q, Qadri F, Kansal R, Rasko DA, Sheikh A, Fleckenstein JM. Conservation and Immunogenicity of Novel Antigens in Diverse Isolates of Enterotoxigenic Escherichia Coli. PloS Negl Trop Dis (2015) 9(1): e0003446. doi: 10.1371/journal.pntd.0003446

41. von Mentzer A, Blackwell GA, Pickard D, Boinett CJ, Joffre E, Page AJ, et al. Long-Read-Sequenced Reference Genomes of the Seven Major Lineages of Enterotoxigenic Escherichia Coli (ETEC) Circulating in Modern Time. Sci Rep (2021) 11(1):9256. doi: 10.1038/s41598-021-88316-2

42. Kuhlmann FM, Laine RO, Afrin S, Nakajima R, Akhtar M, Vickers T, et al. Contribution of Noncanonical Antigens to Virulence and Adaptive Immunity in Human Infection With Enterotoxigenic E. Coli. Infect Immun (2021) 89(5):e00004121. doi: 10.1101/2020.10.20.348110

43. Baldi DL, Higginson EE, Hocking DM, Praszkier J, Cavaliere R, James CE, et al. The Type II Secretion System and Its Ubiquitous Lipoprotein Substrate, Ssle, Are Required for Biofilm Formation and Virulence of Enteropathogenic Escherichia Coli. Infect Immun (2012) 80(6):2042-52. doi: 10.1128/ IAI.06160-11

44. Luo Q, Kumar P, Vickers TJ, Sheikh A, Lewis WG, Rasko DA, et al. Enterotoxigenic Escherichia Coli Secretes a Highly Conserved MucinDegrading Metalloprotease to Effectively Engage Intestinal Epithelial Cells. Infect Immun (2014) 82(2):509-21. doi: 10.1128/IAI.01106-13

45. Nesta B, Valeri M, Spagnuolo A, Rosini R, Mora M, Donato P, et al. Ssle Elicits Functional Antibodies That Impair In Vitro Mucinase Activity and In Vivo Colonization by Both Intestinal and Extraintestinal Escherichia Coli Strains. PloS Pathog (2014) 10(5):e1004124. doi: 10.1371/journal.ppat. 1004124

46. Naili I, Vinot J, Baudner BC, Bernalier-Donadille A, Pizza M, Desvaux M, et al. Mixed Mucosal-Parenteral Immunizations With the Broadly Conserved Pathogenic Escherichia Coli Antigen Ssle Induce a Robust Mucosal and Systemic Immunity Without Affecting the Murine Intestinal Microbiota. Vaccine (2019) 37(2):314-24. doi: 10.1016/j.vaccine.2018.10.008

47. Rasko DA, Rosovitz MJ, Myers GS, Mongodin EF, Fricke WF, Gajer P, et al. The Pangenome Structure of Escherichia Coli: Comparative Genomic Analysis of E. Coli Commensal and Pathogenic Isolates. J Bacteriol (2008) 190(20):6881-93. doi: 10.1128/JB.00619-08

48. Wolf MK. Occurrence, Distribution, and Associations of O and H Serogroups, Colonization Factor Antigens, and Toxins of Enterotoxigenic Escherichia Coli. Clin Microbiol Rev (1997) 10(4):569-84. doi: 10.1128/CMR.10.4.569

49. Iguchi A, von Mentzer A, Kikuchi T, Thomson NR. An Untypeable Enterotoxigenic Escherichia Coli Represents One of the Dominant Types Causing Human Disease. Microb Genom (2017) 3(9):e000121. doi: 10.1099/ mgen.0.000121

50. Mottram L, Chakraborty S, Cox E, Fleckenstein J. How Genomics can be Used to Understand Host Susceptibility to Enteric Infection, Aiding in the Development of Vaccines and Immunotherapeutic Interventions. Vaccine (2019) 37(34):4805-10. doi: 10.1016/j.vaccine.2019.01.016
51. Argimon S, Abudahab K, Goater RJE, Fedosejev A, Bhai J, Glasner C, et al. Microreact: Visualizing and Sharing Data for Genomic Epidemiology and Phylogeography. Microb Genom (2016) 2(11):e000093. doi: 10.1099/ mgen.0.000093

52. von Mentzer A, Connor TR, Wieler LH, Semmler T, Iguchi A, Thomson NR, et al. Identification of Enterotoxigenic Escherichia Coli (ETEC) Clades With Long-Term Global Distribution. Nat Genet (2014) 46(12):1321-6. doi: 10.1038/ng.3145

53. Sahl JW, Steinsland H, Redman JC, Angiuoli SV, Nataro JP, Sommerfelt H, et al. A Comparative Genomic Analysis of Diverse Clonal Types of Enterotoxigenic Escherichia Coli Reveals Pathovar-Specific Conservation. Infect Immun (2011) 79(2):950-60. doi: 10.1128/IAI.00932-10

54. Sahl JW, Sistrunk JR, Baby NI, Begum Y, Luo Q, Sheikh A, et al. Insights Into Enterotoxigenic Escherichia Coli Diversity in Bangladesh Utilizing Genomic Epidemiology. Sci Rep (2017) 7(1):3402. doi: 10.1038/s41598-017-03631-x

55. Chakraborty S, Randall A, Vickers TJ, Molina D, Harro CD, DeNearing B, et al. Human Experimental Challenge With Enterotoxigenic Escherichia Coli Elicits Immune Responses to Canonical and Novel Antigens Relevant to Vaccine Development. J Infect Dis (2018) 218(9):1436-46. doi: 10.1093/ infdis/jiy 312

56. Chakraborty S, Randall A, Vickers TJ, Molina D, Harro CD, DeNearing B, et al. Interrogation of a Live-Attenuated Enterotoxigenic Escherichia Coli Vaccine Highlights Features Unique to Wild-Type Infection. NPJ Vaccines (2019) 4:37. doi: 10.1038/s41541-019-0131-7

57. Lopez-Vidal Y, Calva JJ, Trujillo A, Ponce de Leon A, Ramos A, Svennerholm AM, et al. Enterotoxins and Adhesins of Enterotoxigenic Escherichia Coli: Are They Risk Factors for Acute Diarrhea in the Community? J Infect Dis (1990) 162(2):442-7. doi: 10.1093/infdis/162.2.442

58. Plotkin SA, Gilbert PB. Nomenclature for Immune Correlates of Protection After Vaccination. Clin Infect Dis (2012) 54(11):1615-7. doi: 10.1093/cid/ cis 238

59. Rao MR, Wierzba TF, Savarino SJ, Abu-Elyazeed R, El-Ghoreb N, Hall ER, et al. Serologic Correlates of Protection Against Enterotoxigenic Escherichia Coli Diarrhea. J Infect Dis (2005) 191(4):562-70. doi: 10.1086/427662

60. Holmgren J, Parashar UD, Plotkin S, Louis J, Ng SP, Desauziers E, et al. Correlates of Protection for Enteric Vaccines. Vaccine (2017) 35(26):335563. doi: 10.1016/j.vaccine.2017.05.005

61. Rogawski McQuade ET, Liu J, Kang G, Kosek MN, Lima AAM, Bessong PO, et al. Protection From Natural Immunity Against Enteric Infections and Etiology-Specific Diarrhea in a Longitudinal Birth Cohort. J Infect Dis (2020) 222(11):1858-68. doi: 10.1093/infdis/jiaa031

62. Lopman BA, Baker JM. Wading Into the Morass: Natural Immunity to Enteropathogens. J Infect Dis (2020) 222(11):1764-7. doi: 10.1093/infdis/ jiaa033

63. Steinsland H, Valentiner-Branth P, Gjessing HK, Aaby P, Molbak K, Sommerfelt H. Protection From Natural Infections With Enterotoxigenic Escherichia Coli: Longitudinal Study. Lancet (2003) 362(9380):286-91. doi: 10.1016/S0140-6736(03)13971-2

64. Cravioto A, Reyes RE, Trujillo F, Uribe F, Navarro A, de la Roca JM, et al. Risk of Diarrhea During the First Year of Life Associated With Initial and Subsequent Colonization by Specific Enteropathogens. Am J Epidemiol (1990) 131(5):886-904. doi: 10.1093/oxfordjournals.aje.a115579

65. Levine MM, Nalin DR, Hoover DL, Bergquist EJ, Hornick RB, Young CR. Immunity to Enterotoxigenic Escherichia Coli. Infect Immun (1979) 23 (3):729-36. doi: 10.1128/iai.23.3.729-736.1979

66. Mani S, Toapanta FR, McArthur MA, Qadri F, Svennerholm AM, Devriendt B, et al. Role of Antigen Specific T and B Cells in Systemic and Mucosal Immune Responses in ETEC and Shigella Infections, and Their Potential to Serve as Correlates of Protection in Vaccine Development. Vaccine (2019) 37 (34):4787-93. doi: 10.1016/j.vaccine.2019.03.040

67. Plotkin SA. Correlates of Protection Induced by Vaccination. Clin Vaccine Immunol (2010) 17(7):1055-65. doi: 10.1128/CVI.00131-10

68. McArthur MA, Chen WH, Magder L, Levine MM, Sztein MB. Impact of CD4+ T Cell Responses on Clinical Outcome Following Oral Administration of Wild-Type Enterotoxigenic Escherichia Coli in Humans. PloS Negl Trop Dis (2017) 11(1):e0005291. doi: 10.1371/journal.pntd.0005291

69. Alam MM, Aktar A, Afrin S, Rahman MA, Aktar S, Uddin T, et al. AntigenSpecific Memory B-Cell Responses to Enterotoxigenic Escherichia Coli 
Infection in Bangladeshi Adults. PloS Negl Trop Dis (2014) 8(4):e2822. doi: 10.1371/journal.pntd.0002822

70. Lundgren A, Jertborn M, Svennerholm AM. Induction of Long Term Mucosal Immunological Memory in Humans by an Oral Inactivated Multivalent Enterotoxigenic Escherichia Coli Vaccine. Vaccine (2016) 34 (27):3132-40. doi: 10.1016/j.vaccine.2016.04.055

71. Cardeno A, Magnusson MK, Quiding-Jarbrink M, Lundgren A. Activated T Follicular Helper-Like Cells Are Released Into Blood After Oral Vaccination and Correlate With Vaccine Specific Mucosal B-Cell Memory. Sci Rep (2018) 8(1):2729. doi: 10.1038/s41598-018-20740-3

72. Leach S, Lundgren A, Carlin N, Lofstrand M, Svennerholm AM. CrossReactivity and Avidity of Antibody Responses Induced in Humans by the Oral Inactivated Multivalent Enterotoxigenic Escherichia Coli (ETEC) Vaccine ETVAX. Vaccine (2017) 35(32):3966-73. doi: 10.1016/ j.vaccine.2017.06.006

73. Qadri F, Akhtar M, Bhuiyan TR, Chowdhury MI, Ahmed T, Rafique TA, et al. Safety and Immunogenicity of the Oral, Inactivated, Enterotoxigenic Escherichia Coli Vaccine ETVAX in Bangladeshi Children and Infants: A Double-Blind, Randomised, Placebo-Controlled Phase 1/2 Trial. Lancet Infect Dis (2020) 20(2):208-19. doi: 10.1016/S1473-3099(19)30571-7

74. Akhtar M, Chowdhury MI, Bhuiyan TR, Kaim J, Ahmed T, Rafique TA, et al. Evaluation of the Safety and Immunogenicity of the Oral Inactivated Multivalent Enterotoxigenic Escherichia Coli Vaccine ETVAX in Bangladeshi Adults in a Double-Blind, Randomized, Placebo-Controlled Phase I Trial Using Electrochemiluminescence and ELISA Assays for Immunogenicity Analyses. Vaccine (2019) 37(37):5645-56. doi: 10.1016/ j.vaccine.2018.11.040

75. Darsley MJ, Chakraborty S, Denearing B, Sack DA, Feller A, Buchwaldt C, et al. ACE527 Oral, Live Attenuated ETEC Vaccine Reduces the Incidence and Severity of Diarrhea in a Human Challenge Model of Diarrheal Disease. Clin Vaccine Immunol (2012) 19(12):1921-31. doi: 10.1128/CVI.00364-12

76. Harro C, Louis Bourgeois A, Sack D, Walker R, DeNearing B, Brubaker J, et al. Live Attenuated Enterotoxigenic Escherichia Coli (ETEC) Vaccine With Dmlt Adjuvant Protects Human Volunteers Against Virulent Experimental ETEC Challenge. Vaccine (2019) 37(14):1978-86. doi: 10.1016/j.vaccine.2019.02.025

77. Brubaker J, Zhang X, Bourgeois AL, Harro C, Sack DA, Chakraborty S. Intestinal and Systemic Inflammation Induced by Symptomatic and Asymptomatic Enterotoxigenic E. Coli Infection and Impact on Intestinal Colonization and ETEC Specific Immune Responses in an Experimental Human Challenge Model. Gut Microbes (2021) 13(1):1-13. doi: 10.1080/ 19490976.2021.1891852

78. Cua DJ, Tato CM. Innate IL-17-Producing Cells: The Sentinels of the Immune System. Nat Rev Immunol (2010) 10(7):479-89. doi: 10.1038/ nri2800

79. Brereton CF, Sutton CE, Ross PJ, Iwakura Y, Pizza M, Rappuoli R, et al. Escherichia Coli Heat-Labile Enterotoxin Promotes Protective Th17 Responses Against Infection by Driving Innate IL-1 and IL-23 Production. J Immunol (2011) 186(10):5896-906. doi: 10.4049/jimmunol.1003789

80. Mitsdoerffer M, Lee Y, Jager A, Kim HJ, Korn T, Kolls JK, et al. Proinflammatory T Helper Type 17 Cells Are Effective B-Cell Helpers. Proc Natl Acad Sci USA (2010) 107(32):14292-7. doi: 10.1073/pnas. 1009234107

81. Clements JD, Norton EB. The Mucosal Vaccine Adjuvant LT(R192G/L211A) or Dmlt. mSphere (2018) 3(4):e00215-18. doi: 10.1128/mSphere.00215-18

82. Barry EM, Levine MM. A Tale of Two Bacterial Enteropathogens and One Multivalent Vaccine. Cell Microbiol (2019) 21(11):e13067. doi: 10.1111/ cmi.13067

83. El-Kamary SS, Cohen MB, Bourgeois AL, Van De Verg L, Bauers N, Reymann M, et al. Safety and Immunogenicity of a Single Oral Dose of Recombinant Double Mutant Heat-Labile Toxin Derived From Enterotoxigenic Escherichia Coli. Clin Vaccine Immunol (2013) 20 (11):1764-70. doi: 10.1128/CVI.00464-13

84. Bernstein DI, Pasetti MF, Brady R, Buskirk AD, Wahid R, Dickey M, et al. A Phase 1 Dose Escalating Study of Double Mutant Heat-Labile Toxin LTR192G/L211A (Dmlt) From Enterotoxigenic Escherichia Coli (ETEC) by Sublingual or Oral Immunization. Vaccine (2019) 37(4):602-11. doi: 10.1016/j.vaccine.2018.12.011
85. Seo H, Lu T, Nandre RM, Duan Q, Zhang W. Immunogenicity Characterization of Genetically Fused or Chemically Conjugated HeatStable Toxin Toxoids of Enterotoxigenic Escherichia Coli in Mice and Pigs. FEMS Microbiol Lett (2019) 366(4):fnz037. doi: 10.1093/femsle/fnz037

86. Barry E, Cassels F, Riddle M, Walker R, Wierzba T. Vaccines Against Shigella and Enterotoxigenic Escherichia Coli: A Summary of the 2018 VASE Conference. Vaccine (2019) 37(34):4768-74. doi: 10.1016/j.vaccine. 2019.02.070

87. Riddle MS, Maciel MJr., Porter CK, Poole ST, Gutierrez RL, Gormley R, et al. A First in Human Clinical Trial Assessing the Safety and Immunogenicity of Transcutaneously Delivered Enterotoxigenic Escherichia Coli Fimbrial Tip Adhesin With Heat-Labile Enterotoxin With Mutation R192G. Vaccine (2020) 38(45):7040-8. doi: 10.1016/j.vaccine.2020.09.025

88. Poole ST, Maciel M Jr, Dinadayala P, Dori KE, McVeigh AL, Liu Y, et al. Biochemical and Immunological Evaluation of Recombinant CS6-Derived Subunit Enterotoxigenic Escherichia Coli Vaccine Candidates. Infect Immun (2019) 87(3):e00788-18. doi: 10.1128/IAI.00788-18

89. O’Dowd A, Maciel M Jr, Poole ST, Jobling MG, Rollenhagen JE, Woods CM, et al. Evaluation of the Immunogenicity and Protective Efficacy of an Enterotoxigenic Escherichia Coli CFA/I Adhesin-Heat-Labile Toxin Chimera. Infect Immun (2020) 88(11):e00252-20. doi: 10.1128/IAI.00252-20

90. Seo H, Lu T, Mani S, Bourgeois AL, Walker R, Sack DA, et al. Adjuvant Effect of Enterotoxigenic Escherichia Coli (ETEC) Double-Mutant HeatLabile Toxin (Dmlt) on Systemic Immunogenicity Induced by the CFA/I/II/ IV MEFA ETEC Vaccine: Dose-Related Enhancement of Antibody Responses to Seven ETEC Adhesins (CFA/I, CS1-CS6). Hum Vaccin Immunother (2020) 16(2):419-25. doi: 10.1080/21645515.2019.1649555

91. Lundgren A, Bourgeois L, Carlin N, Clements J, Gustafsson B, Hartford M, et al. Safety and Immunogenicity of an Improved Oral Inactivated Multivalent Enterotoxigenic Escherichia Coli (ETEC) Vaccine Administered Alone and Together With Dmlt Adjuvant in a DoubleBlind, Randomized, Placebo-Controlled Phase I Study. Vaccine (2014) 32 (52):7077-84. doi: 10.1016/j.vaccine.2014.10.069

92. Holmgren J, Bourgeois L, Carlin N, Clements J, Gustafsson B, Lundgren A, et al. Development and Preclinical Evaluation of Safety and Immunogenicity of an Oral ETEC Vaccine Containing Inactivated E. Coli Bacteria Overexpressing Colonization Factors CFA/I, CS3, CS5 and CS6 Combined With a Hybrid LT/CT B Subunit Antigen, Administered Alone and Together With Dmlt Adjuvant. Vaccine (2013) 31(20):2457-64. doi: 10.1016/ j.vaccine.2013.03.027

93. Harro C, Sack D, Bourgeois AL, Walker R, Denearing B, Feller A, et al. A Combination Vaccine Consisting of Three Live Attenuated Enterotoxigenic Escherichia Coli Strains Expressing a Range of Colonization Factors and LTB Is Well Tolerated and Immunogenic in a Placebo-Controlled Double-Blind Phase I Trial in Healthy Adults. Clin Vaccine Immunol (2011) 18(12):211827. doi: 10.1128/CVI.05342-11

94. Vidal RM, Muhsen K, Tennant SM, Svennerholm AM, Sow SO, Sur D, et al. Colonization Factors Among Enterotoxigenic Escherichia Coli Isolates From Children With Moderate-to-Severe Diarrhea and From Matched Controls in the Global Enteric Multicenter Study (GEMS). PloS Negl Trop Dis (2019) 13 (1):e0007037. doi: 10.1371/journal.pntd.0007037

95. Peruski LF Jr, Kay BA, El-Yazeed RA, El-Etr SH, Cravioto A, Wierzba TF, et al. Phenotypic Diversity of Enterotoxigenic Escherichia Coli Strains From a Community-Based Study of Pediatric Diarrhea in Periurban Egypt. J Clin Microbiol (1999) 37(9):2974-8. doi: 10.1128/JCM.37.9.2974-2978.1999

96. Begum YA, Baby NI, Faruque AS, Jahan N, Cravioto A, Svennerholm AM, et al. Shift in Phenotypic Characteristics of Enterotoxigenic Escherichia Coli (ETEC) Isolated From Diarrheal Patients in Bangladesh. PloS Negl Trop Dis (2014) 8(7):e3031. doi: 10.1371/journal.pntd.0003031

97. Rockabrand DM, Shaheen HI, Khalil SB, Peruski LF Jr, Rozmajzl PJ, Savarino SJ, et al. Enterotoxigenic Escherichia Coli Colonization Factor Types Collected From 1997 to 2001 in US Military Personnel During Operation Bright Star in Northern Egypt. Diagn Microbiol Infect Dis (2006) 55(1):9-12. doi: 10.1016/j.diagmicrobio.2005.12.002

98. Shaheen HI, Abdel Messih IA, Klena JD, Mansour A, El-Wakkeel Z, Wierzba TF, et al. Phenotypic and Genotypic Analysis of Enterotoxigenic Escherichia Coli in Samples Obtained From Egyptian Children Presenting to Referral Hospitals. J Clin Microbiol (2009) 47(1):189-97. doi: 10.1128/JCM.01282-08 
99. Khalil I, Walker R, Porter CK, Muhib F, Chilengi R, Cravioto A, et al. Enterotoxigenic Escherichia Coli (ETEC) Vaccines: Priority Activities to Enable Product Development, Licensure, and Global Access. Vaccine (2021) 39(31):4266-77. doi: 10.1016/j.vaccine.2021.04.018

100. Turner AK, Stephens JC, Beavis JC, Greenwood J, Gewert C, Randall R, et al. Generation and Characterization of a Live Attenuated Enterotoxigenic Escherichia Coli Combination Vaccine Expressing Six Colonization Factors and Heat-Labile Toxin Subunit B. Clin Vaccine Immunol (2011) 18 (12):2128-35. doi: 10.1128/CVI.05345-11

101. Kotloff KL, Simon JK, Pasetti MF, Sztein MB, Wooden SL, Livio S, et al. Safety and Immunogenicity of CVD 1208S, a Live, Oral Deltaguaba Deltasen Deltaset Shigella Flexneri 2a Vaccine Grown on Animal-Free Media. Hum Vaccin (2007) 3(6):268-75. doi: 10.4161/hv.4746

102. Medeiros P, Bolick DT, Ledwaba SE, Kolling GL, Costa DVS, Oria RB, et al. A Bivalent Vaccine Confers Immunogenicity and Protection Against Shigella Flexneri and Enterotoxigenic Escherichia Coli Infections in Mice. NPJ Vaccines (2020) 5:30. doi: 10.1038/s41541-020-0180-y

103. Clemens JD, Sack DA, Harris JR, Chakraborty J, Neogy PK, Stanton B, et al. Cross-Protection by B Subunit-Whole Cell Cholera Vaccine Against Diarrhea Associated With Heat-Labile Toxin-Producing Enterotoxigenic Escherichia Coli: Results of a Large-Scale Field Trial. J Infect Dis (1988) 158(2):372-7. doi: 10.1093/infdis/158.2.372

104. McKenzie R, Bourgeois AL, Frech SA, Flyer DC, Bloom A, Kazempour K, et al. Transcutaneous Immunization With the Heat-Labile Toxin (LT) of Enterotoxigenic Escherichia Coli (ETEC): Protective Efficacy in a DoubleBlind, Placebo-Controlled Challenge Study. Vaccine (2007) 25(18):3684-91. doi: 10.1016/j.vaccine.2007.01.043

105. Frech SA, Dupont HL, Bourgeois AL, McKenzie R, Belkind-Gerson J, Figueroa JF, et al. Use of a Patch Containing Heat-Labile Toxin From Escherichia Coli Against Travellers' Diarrhoea: A Phase II, Randomised, Double-Blind, Placebo-Controlled Field Trial. Lancet (2008) 371 (9629):2019-25. doi: 10.1016/S0140-6736(08)60839-9

106. Behrens RH, Cramer JP, Jelinek T, Shaw H, von Sonnenburg F, Wilbraham D, et al. Efficacy and Safety of a Patch Vaccine Containing Heat-Labile Toxin From Escherichia Coli Against Travellers' Diarrhoea: A Phase 3, Randomised, Double-Blind, Placebo-Controlled Field Trial in Travellers From Europe to Mexico and Guatemala. Lancet Infect Dis (2013) 14 (3):197-204. doi: 10.1016/S1473-3099(13)70297-4

107. Riddle MS, Savarino SJ. Moving Beyond a Heat-Labile Enterotoxin-Based Vaccine Against Enterotoxigenic Escherichia Coli. Lancet Infect Dis (2014) 14 (3):174-5. doi: 10.1016/S1473-3099(13)70355-4

108. Sixma TK, Kalk KH, van Zanten BA, Dauter Z, Kingma J, Witholt B, et al. Refined Structure of Escherichia Coli Heat-Labile Enterotoxin, a Close Relative of Cholera Toxin. J Mol Biol (1993) 230(3):890-918. doi: 10.1006/jmbi.1993.1209

109. Ozaki H, Sato T, Kubota H, Hata Y, Katsube Y, Shimonishi Y. Molecular Structure of the Toxin Domain of Heat-Stable Enterotoxin Produced by a Pathogenic Strain of Escherichia Coli. A Putative Binding Site for a Binding Protein on Rat Intestinal Epithelial Cell Membranes. J Biol Chem (1991) 266(9):5934-41.

110. Shimamoto S, Fukutsuji M, Osumi T, Goto M, Toyoda H, Hidaka Y. Topological Regulation of the Bioactive Conformation of a Disulfide-Rich Peptide, Heat-Stable Enterotoxin. Molecules (2020) 25(20):4798. doi: $10.3390 /$ molecules 25204798

111. Pettersen EF, Goddard TD, Huang CC, Meng EC, Couch GS, Croll TI, et al. UCSF Chimerax: Structure Visualization for Researchers, Educators, and Developers. Protein Sci (2021) 30(1):70-82. doi: 10.1002/pro.3943

112. Bryant AP, Busby RW, Bartolini WP, Cordero EA, Hannig G, Kessler MM, et al. Linaclotide Is a Potent and Selective Guanylate Cyclase C Agonist That Elicits Pharmacological Effects Locally in the Gastrointestinal Tract. Life Sci (2010) 86(19-20):760-5. doi: 10.1016/j.lfs.2010.03.015

113. Taxt AM, Diaz Y, Bacle A, Grauffel C, Reuter N, Aasland R, et al. Characterization of Immunological Cross-Reactivity Between Enterotoxigenic Escherichia Coli Heat-Stable Toxin and Human Guanylin and Uroguanylin. Infect Immun (2014) 82(7):2913-22. doi: 10.1128/IAI.01749-14

114. Diaz Y, Govasli ML, Zegeye ED, Sommerfelt H, Steinsland H, Puntervoll P. Immunizations With Enterotoxigenic Escherichia Coli Heat-Stable Toxin Conjugates Engender Toxin-Neutralizing Antibodies in Mice That Also Cross-React With Guanylin and Uroguanylin. Infect Immun (2019) 87(7): e00099-19. doi: 10.1128/IAI.00099-19
115. Puntervoll P, Clements JD, Diaz Y, Nataro JP, Taxt AM, Zhang W, et al. eds. Rational Design of a Vaccine Against the Heat-Stable Toxin of Enterotoxigenic Escherichia Coli. Bangkok, Thailand: Vaccines for Enteric Diseases (2013).

116. Taxt A, Aasland R, Sommerfelt H, Nataro J, Puntervoll P. Heat-Stable Enterotoxin of Enterotoxigenic Escherichia Coli as a Vaccine Target. Infection Immun (2010) 78(5):1824-31. doi: 10.1128/IAI.01397-09

117. Taxt AM, Diaz Y, Aasland R, Clements JD, Nataro JP, Sommerfelt H, et al. Towards Rational Design of a Toxoid Vaccine Against the Heat-Stable Toxin of Escherichia Coli. Infect Immun (2016) 84(4):1239-49. doi: 10.1128/ IAI.01225-15

118. Govasli ML, Diaz Y, Puntervoll P. Virus-Like Particle-Display of the Enterotoxigenic Escherichia Coli Heat-Stable Toxoid Sth-A14T Elicits Neutralizing Antibodies in Mice. Vaccine (2019) 37(43):6405-14. doi: 10.1016/j.vaccine.2019.09.004

119. Fleckenstein JM. Providing Structure to Enterotoxigenic Escherichia Coli Vaccine Development. J Infect Dis (2017) 216(1):1-3. doi: 10.1093/infdis/jix146

120. Savarino SJ, McKenzie R, Tribble DR, Porter CK, O’Dowd A, Cantrell JA, et al. Prophylactic Efficacy of Hyperimmune Bovine Colostral Antiadhesin Antibodies Against Enterotoxigenic Escherichia Coli Diarrhea: A Randomized, Double-Blind, Placebo-Controlled, Phase 1 Trial. J Infect Dis (2017) 216(1):7-13. doi: 10.1093/infdis/jix144

121. Poncet D, Hessler C, Liang H, Gautheron S, Sergent M, Rintala ND, et al. Preclinical Optimization of an Enterotoxigenic Escherichia Coli Adjuvanted Subunit Vaccine Using Response Surface Design of Experiments. NPJ Vaccines (2020) 5:83. doi: 10.1038/s41541-020-00228-w

122. Talaat KR, Porter CK, Bourgeois AL, Lee TK, Duplessis CA, Maciel MJr., et al. Oral Delivery of Hyperimmune Bovine Serum Antibodies Against CS6-Expressing Enterotoxigenic Escherichia Coli as a Prophylactic Against Diarrhea. Gut Microbes (2020) 12(1):1732852. doi: 10.1080/19490976.2020.1732852

123. Liu Y, Shahabudin S, Farid S, Lee LH, McVeigh AL, Maciel MJr., et al. CrossReactivity, Epitope Mapping, and Potency of Monoclonal Antibodies to Class 5 Fimbrial Tip Adhesins of Enterotoxigenic Escherichia Coli. Infect Immun (2020) 88(11):e00246-20. doi: 10.1128/IAI.00246-20

124. Amcheslavsky A, Wallace AL, Ejemel M, Li Q, McMahon CT, Stoppato M, et al. Anti-Cfae Nanobodies Provide Broad Cross-Protection Against Major Pathogenic Enterotoxigenic Escherichia Coli Strains, With Implications for Vaccine Design. Sci Rep (2021) 11(1):2751. doi: 10.1038/s41598-021-81895-0

125. Seo H, Garcia C, Ruan X, Duan Q, Sack DA, Zhang W. Preclinical Characterization of Immunogenicity and Efficacy Against Diarrhea From Mecvax, A Multivalent Enterotoxigenic E. Coli Vaccine Candidate. Infect Immun (2021). doi: 10.1128/IAI.00106-21

126. WHO. DRAFT WHO Preferred Product Characteristics for Vaccines Against Enterotoxigenic Escherichia Coli 2020. Available at: https://www.who.int/ immunization/research/ppc-tpp/PPC_ETEC_April_2020_Public_ Consultation.pdf?ua $=1$.

127. Hanevik K, Chen WH, Talaat KR, Porter C, Bourgeois L. The Way Forward for ETEC Controlled Human Infection Models (Chims). Vaccine (2019) 37 (34):4794-9. doi: 10.1016/j.vaccine.2019.01.003

Author Disclaimer: The opinions expressed here are those of the author and not those of the NIH or the VA.

Conflict of Interest: The author declares that the research was conducted in the absence of any commercial or financial relationships that could be construed as a potential conflict of interest.

Publisher's Note: All claims expressed in this article are solely those of the authors and do not necessarily represent those of their affiliated organizations, or those of the publisher, the editors and the reviewers. Any product that may be evaluated in this article, or claim that may be made by its manufacturer, is not guaranteed or endorsed by the publisher.

Copyright $\odot 2021$ Fleckenstein. This is an open-access article distributed under the terms of the Creative Commons Attribution License (CC BY). The use, distribution or reproduction in other forums is permitted, provided the original author(s) and the copyright owner(s) are credited and that the original publication in this journal is cited, in accordance with accepted academic practice. No use, distribution or reproduction is permitted which does not comply with these terms. 